\title{
Erratum
}

\section{Boundary Scattering, Symmetric Spaces and the Principal Chiral Model on the Half-Line}

\author{
N.J. MacKay, B.J. Short \\ Department of Mathematics, University of York, York YO10 5DD, U.K.
}

Received: 29 July 2003 / Accepted: 3 November 2003

Erratum published online: 29 January 2004 - ㅇ Springer-Verlag 2004

Commun. Math. Phys. 233, 313-354 (2003)

In the above article, two points require correction. First, the discussion regarding charge conjugation in Sect. 3.2.2 is unnecessary and may be replaced by an appeal only to crossing unitarity. Consequently (3.16) should now read

$$
\begin{array}{cll}
E^{\dagger} E=I & \text { and } & \operatorname{det} E=1, \\
\rho(\theta)=\rho\left(-\theta^{*}\right)^{*} & \text { and } & \rho(\theta) \rho(-\theta)=1 .
\end{array}
$$

Second, the crossing unitarity relation

$$
K^{i j}\left(\frac{i \pi}{2}-\theta\right)=S_{\bar{j} \bar{k}}^{i l}(2 \theta) K^{l k}\left(\frac{i \pi}{2}+\theta\right)
$$

was incorrectly stated, and should be replaced by

$$
K^{i j}\left(\frac{i \pi}{2}-\theta\right)=S_{\overline{j k}}^{i \bar{l}}(2 \theta) K^{\bar{l} \bar{k}}\left(\frac{i \pi}{2}+\theta\right) .
$$

These corrections result in some changes to the allowed $K$-matrices. We give here only the list of the final boundary $S$-matrices, which replaces the existing Sect. 4.2:

4.2. The boundary $S$-matrices. First we give a scalar factor which has altered slightly from the original version and also an additional scalar factor:

$$
\begin{aligned}
& \mu(\theta)=\frac{1}{4 \pi^{2} c^{2}} \\
& \times \frac{\Gamma\left(\frac{-\theta}{2 i \pi}+\frac{1}{2}+\frac{1}{2 i \pi c}\right) \Gamma\left(\frac{-\theta}{2 i \pi}+\frac{1}{2}-\frac{1}{2 i \pi c}\right) \Gamma\left(\frac{\theta}{2 i \pi}+\frac{1}{2 i \pi c}\right) \Gamma\left(\frac{\theta}{2 i \pi}-\frac{1}{2 i \pi c}\right)}{\Gamma\left(\frac{\theta}{2 i \pi}+\frac{1}{2}+\frac{1}{2 i \pi c}\right) \Gamma\left(\frac{\theta}{2 i \pi}+\frac{1}{2}-\frac{1}{2 i \pi c}\right) \Gamma\left(\frac{-\theta}{2 i \pi}+1+\frac{1}{2 i \pi c}\right) \Gamma\left(\frac{-\theta}{2 i \pi}+1-\frac{1}{2 i \pi c}\right)},
\end{aligned}
$$


$\lambda(\theta)=\frac{1}{4 \pi^{2} c^{2}}$

$\frac{\Gamma\left(\frac{-\theta}{2 i \pi}+\frac{M}{N}+\frac{1}{2 i \pi c}\right) \Gamma\left(\frac{-\theta}{2 i \pi}+1-\frac{M}{N}-\frac{1}{2 i \pi c}\right) \Gamma\left(\frac{\theta}{2 i \pi}+\frac{1}{2 i \pi c}\right) \Gamma\left(\frac{\theta}{2 i \pi}-\frac{1}{2 i \pi c}\right)}{\Gamma\left(\frac{\theta}{2 i \pi}+\frac{M}{N}+\frac{1}{2 i \pi c}\right) \Gamma\left(\frac{\theta}{2 i \pi}+1-\frac{M}{N}-\frac{1}{2 i \pi c}\right) \Gamma\left(\frac{-\theta}{2 i \pi}+1+\frac{1}{2 i \pi c}\right) \Gamma\left(\frac{-\theta}{2 i \pi}+1-\frac{1}{2 i \pi c}\right)}$,

in both of which $c$ is a freely varying parameter unless otherwise stated below.

The PCM $S$-matrices may then be written as follows.

4.2.1. $S U(N)$. We have found two types of boundary $S$-matrix for $S U(N)$,

$$
K_{P C M}(\theta)=-(1-h)_{\theta} \mu(\theta)\left(v(\theta)\left(I+c \theta E_{L}\right) \otimes v(\theta)\left(I+c \theta E_{R}\right)\right)
$$

(whose $c \rightarrow \infty$ limit:

$$
K_{P C M}(\theta)=-(1-h)_{\theta}\left(\eta(\theta) E_{L} \otimes \eta(\theta) E_{R}\right)
$$

is a valid PCM boundary scattering matrix) where

$$
E_{L / R} \in \frac{S U(N)}{S(U(N / 2) \times U(N / 2))},
$$

and

$$
K_{P C M}(\theta)=-(1-h)_{\theta} \lambda(\theta)\left(v(\theta)\left(I+c \theta E_{L}\right) \otimes v(\theta)\left(I+c \theta E_{R}\right)\right),
$$

where

$$
E_{L / R} \in \frac{S U(N)}{S(U(N-M) \times U(M))} .
$$

4.2.2. $S O(N)$. For $S O(N)$ three types have been found,

$$
\begin{aligned}
K_{P C M}(\theta)= & -\left(\frac{h}{2}+2\right)_{\theta}\left(\frac{h}{2}+1\right)_{\theta}(1-h)_{\theta} \mu(\theta) \\
& \times\left(v(\theta) \epsilon_{3,3}(\theta)\left(I+c \theta E_{L}\right) \otimes v(\theta) \epsilon_{3,3}(\theta)\left(I+c \theta E_{R}\right)\right)
\end{aligned}
$$

(whose $c \rightarrow \infty$ limit:

$$
K_{P C M}(\theta)=-\left(\frac{h}{2}+2\right)_{\theta}\left(\frac{h}{2}+1\right)_{\theta}(1-h)_{\theta}\left(\eta(\theta) \epsilon_{3,3}(\theta) E_{L} \otimes \eta(\theta) \epsilon_{3,3}(\theta) E_{R}\right)
$$

is a valid PCM boundary scattering matrix) where

$$
\begin{gathered}
E_{L / R} \in \frac{S O(N)}{U(N / 2)} \times\{+1,-1\}, \\
K_{P C M}(\theta)=-\left(\frac{h}{2}+2\right)_{\theta}\left(\frac{h}{2}+1\right)_{\theta}(1-h)_{\theta}\left(\eta(\theta) \epsilon_{3,1}(\theta) E_{L} \otimes \eta(\theta) \epsilon_{3,1}(\theta) E_{R}\right),
\end{gathered}
$$


where

$$
E_{L / R} \in \frac{S O(N)}{S(O(N / 2) \times O(N / 2))},
$$

and

$$
\begin{aligned}
K_{P C M}(\theta)= & -\left(\frac{h}{2}+2\right)_{\theta}\left(\frac{h}{2}+1\right)_{\theta}(1-h)_{\theta} \mu(\theta) \\
& \times\left(v(\theta) \epsilon_{3,1}(\theta)\left(I+c \theta E_{L}\right) \otimes v(\theta) \epsilon_{3,1}(\theta)\left(I+c \theta E_{R}\right)\right),
\end{aligned}
$$

where

$$
E_{L / R} \in \frac{S O(N)}{S(O(N-M) \times O(M))} \quad \text { and } c=\frac{2 i h}{\pi(2 M-N)} \text { in } \mu(\theta) .
$$

4.2.3. $S p(N)$. Three types of $K_{P C M}(\theta)$ have also been found for $S p(N)$,

$$
\begin{aligned}
K_{P C M}(\theta)= & -\left(\frac{h}{2}+2\right)_{\theta}\left(\frac{h}{2}+1\right)_{\theta}(1-h)_{\theta} \mu(\theta) \\
& \times\left(v(\theta) \epsilon_{3,1}(\theta)\left(I+c \theta E_{L}\right) \otimes v(\theta) \epsilon_{3,1}(\theta)\left(I+c \theta E_{R}\right)\right)
\end{aligned}
$$

(whose $c \rightarrow \infty$ limit:

$$
K_{P C M}(\theta)=-\left(\frac{h}{2}+2\right)_{\theta}\left(\frac{h}{2}+1\right)_{\theta}(1-h)_{\theta}\left(\eta(\theta) \epsilon_{3,1}(\theta) E_{L} \otimes \eta(\theta) \epsilon_{3,1}(\theta) E_{R}\right)
$$

is a valid PCM boundary scattering matrix) where

$$
\begin{gathered}
E_{L / R} \in \frac{S p(N)}{U(N / 2)}, \\
K_{P C M}(\theta)=-\left(\frac{h}{2}+2\right)_{\theta}\left(\frac{h}{2}+1\right)_{\theta}(1-h)_{\theta}\left(\eta(\theta) \epsilon_{3,3}(\theta) E_{L} \otimes \eta(\theta) \epsilon_{3,3}(\theta) E_{R}\right),
\end{gathered}
$$

where

$$
E_{L / R} \in \frac{S p(N)}{S p(N / 2) \times S p(N / 2))}
$$

and

$$
\begin{aligned}
K_{P C M}(\theta)= & -\left(\frac{h}{2}+2\right)_{\theta}\left(\frac{h}{2}+1\right)_{\theta}(1-h)_{\theta} \mu(\theta) \\
& \times\left(v(\theta) \epsilon_{3,3}(\theta)\left(I+c \theta E_{L}\right) \otimes v(\theta) \epsilon_{3,3}(\theta)\left(I+c \theta E_{R}\right)\right),
\end{aligned}
$$

where

$$
E_{L / R} \in \frac{S p(N)}{S p(N-M) \times S p(M))} \quad \text { and } c=\frac{2 i h}{\pi(2 M-N)} \text { in } \mu(\theta) .
$$


4.2.4. $S U(N)$-conjugating. Lastly, we have found two types of representationconjugating boundary $S$-matrix for $S U(N)$

$$
K_{P C M}(\theta)=\left(\frac{h}{2}+2\right)_{\theta}\left(\frac{h}{2}+1\right)_{\theta}\left(\epsilon_{1,1}(\theta) E_{L} \otimes \epsilon_{1,1}(\theta) E_{R}\right),
$$

where

$$
E_{L / R} \in \frac{S U(N)}{S O(N)}
$$

and

$$
K_{P C M}(\theta)=\left(\frac{h}{2}+2\right)_{\theta}\left(\frac{h}{2}+1\right)_{\theta}\left(\epsilon_{1,3}(\theta) E_{L} \otimes \epsilon_{1,3}(\theta) E_{R}\right)
$$

where

$$
E_{L / R} \in\left\{1, \omega^{2}\right\} \times \frac{S U(N)}{S p(N)} \quad\left(\omega^{N}=-1\right) .
$$

The discussion and conclusions are not significantly affected.

Communicated by M. Aizenman 